\title{
A $8 \mathrm{~mW} 72 \mathrm{~dB} \Sigma \Delta$-modulator ADC with $2.4 \mathrm{MHz}$ $\mathrm{BW}$ in $130 \mathrm{~nm}$ CMOS
}

M. De Bock, P. Rombouts

This document is an author's draft version submitted for publication in Analog Integrated Circuits And Signal Processing, The actual version was published as:

M. De Bock and P. Rombouts, “A 8 mW 72 dB Sigma Delta-modulator ADC with 2.4 MHz BW in $130 \mathrm{~nm}$ CMOS,” Analog Integrated Circuits And Signal Processing, vol. 72, pp. 541-548, Sept. 2012. 


\title{
A $8 \mathrm{~mW} 72 \mathrm{~dB} \Sigma \Delta$-modulator ADC with $2.4 \mathrm{MHz} \mathrm{BW}$ in $130 \mathrm{~nm}$ CMOS
}

\author{
Maarten De Bock • Pieter Rombouts
}

Received: date / Accepted: date

\begin{abstract}
A double-sampling split $\Sigma \Delta$-ADC with bilinear integrators and a 7-level quantizer is presented. It achieves third order noise shaping with a second order modulator through quantization noise-coupling. The modulator is integrated in a $130 \mathrm{~nm}$ CMOS technology. For a clock frequency of $48 \mathrm{MHz}$ and an oversampling ratio of $20(2.4 \mathrm{MHz}$ signal bandwidth $)$, it achieves $72 \mathrm{~dB}$ DR and $68 \mathrm{~dB}$ SNR. The prototype consumes $8 \mathrm{~mW}$ from a $1.2 \mathrm{~V}$ voltage supply.
\end{abstract}

Keywords Analog-to-digital converters · double sampling $\cdot \Sigma \Delta$-modulation $\cdot$ noise-coupling $\cdot 130 \mathrm{~nm}$ CMOS technology

\section{Introduction}

Wide-band $\Sigma \Delta$-modulation requires a low oversampling ratio (OSR) in combination with high-order noise shaping and multi-bit quantization. In double-sampling $\Sigma \Delta$ modulators, the outputs of the switched capacitor (SC) circuits are updated during both clock phases, doubling the sampling frequency to twice the master clock frequency. Thus for the same power budget, the OSR is doubled. In [1],[2] an enhanced split-architecture $\Sigma \Delta$ ADC is presented. Through the use of noise-coupling the effective noise shaping order is increased. This can lead to a significant reduction in power consumption for low-order modulators [3].

In this paper, we combine double-sampling with cross noise-coupling for a fully integrated prototype.

M. De Bock · P. Rombouts

Department of Electronics and Information Systems

Faculty of Engineering, Ghent University

Sint-Pietersnieuwstraat 41, B-9000 Gent, Belgium

E-mail: maarten.debock@elis.ugent.be

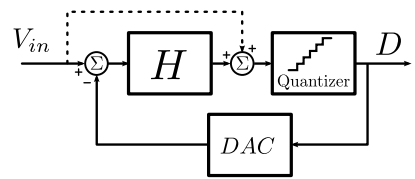

(a)

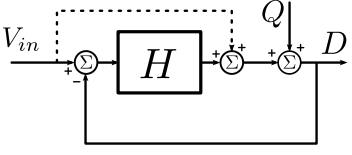

(b)
Fig. 1 (a) A $\Sigma \Delta$-modulator and (b) the linearized model.

Section 3 explains the modulator architecture. The circuit level design is discussed in section 4 . Measurement results for the integrated prototype are presented in section 5 .

\section{System Level Design}

Fig. 1(a) shows the conceptual diagram of a $\Sigma \Delta$ - modulator. The quantizer is embedded in a control loop with loop filter $H$. The digital output $D$ of the quantizer is fed back to the input of the filter via a digital-to-analog converter $(D A C)$. A common analysis for this system is shown in Fig. 1(b). Here, the quantizer is modelled as an additive white noise contribution $Q$ and the $\mathrm{D} / \mathrm{A}$ converter for the feedback path is assumed to be ideal. Fig. 1 also shows an additional feed-forward path from the input signal $V_{i n}$ towards the input of the quantizer (dashed line) [4]. In this case, the digital output $D$ of the complete system can be written as:

$D(z)=V_{\text {in }}(z)+N T F(z) Q(z)$

Here, $N T F(z)$ corresponds to the noise transfer function. The main building block in the loop filter $H$ is an integrator. The magnitude of the loop filter will be high in the low-pass signal band, but low outside the signal band. This way the NTF will be nearly zero in the signal 


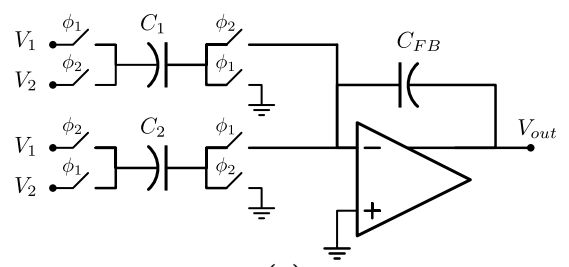

(a)

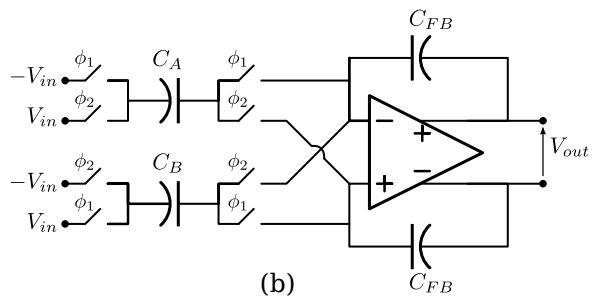

Fig. 2 (a) A conventional and (b) a fully-floating bilinear double-sampling integrator.

band but not outside the signal band. The quantization noise is thus shaped outside the signal band and the output has a very high signal-to-noise-plus-distortion ratio (SNDR) within the signal band.

\subsection{Double-Sampling}

The efficiency of SC-circuits can be doubled easily by using double-sampling techniques. Fig. 2(a) shows a conventional double sampling integrator with 2 input signals $V_{1}$ and $V_{2}$. The capacitors $C_{1}$ and $C_{2}$ are equal. If $V_{2}=-V_{1}$ this circuit performs a bilinear integration:

$V_{\text {out }}=\frac{C_{1}}{C_{F B}} \frac{\left(1+z^{-1}\right)}{\left(1-z^{-1}\right)} V_{1}(z)$

However, the conventional double-sampling integrator shown in Fig. 2(a) will suffer from quantization noise folding due to mismatch between capacitors $C_{1}$ and $C_{2}$. Noise folding will transform signals around the Nyquist frequency $\left(f_{s} / 2\right)$ towards DC, and as such will degrade the performance of the modulator [5]. Fig. 2(b) shows an alternative circuit that performs a bilinear integration (eq. 2) [6]. For this circuit, it can be shown that mismatch between $C_{1}$ and $C_{2}$ will not lead to noise folding.

However, because of the bilinear factor $\left(1+z^{-1}\right)$ in the numerator of eq. (2), the modulator architecture should be modified [7]. The modified architecture for a $2^{\text {nd }}$ order modulator is shown in Fig. 3. The fully floating bilinear integrator is used here for the critical DAC feedback branches, as only this signal path contains signals around the Nyquist frequency. The modulator also shows an additional parameter $b_{3}$. This gives the necessary freedom to design the NTF at will [7].

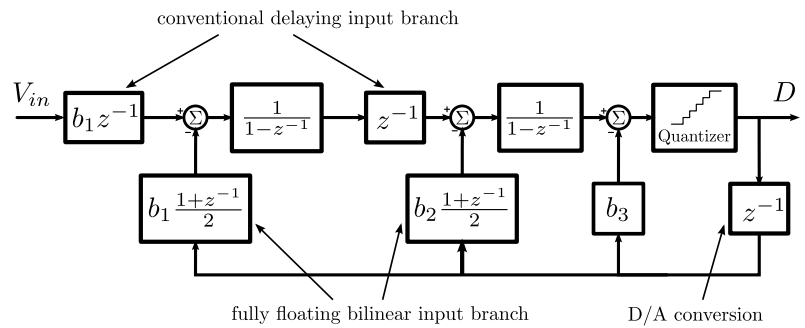

Fig. 3 A modified modulator architecture with bilinear integrators.

\subsection{Cross Noise-Coupling}

In a split architecture $\Sigma \Delta \mathrm{A} / \mathrm{D}$ converter, a single modulator is split into two identical halves (Fig. 4). As the two resulting modulator loops have capacitors half the size of those in the original modulator, the total power dissipation and chip area will essentially remain the same. Each modulator loop processes the same input signal independently of the other loop. The two outputs are then combined to generate the total average output signal $D_{a v}$. Since thermal as well as quantization noise are uncorrelated between the two loops, the SNR performance of the split modulator is the same as for the original single modulator.

The noise added by the quantizer can easily be determined by taking the difference between the output and the input of the quantizer. In $[1,2]$ cross noisecoupled split $\Sigma \Delta$-modulator architectures are presented. In such an architecture, the noise of one loop is injected into the other loop as shown in Fig. 4 (dashed line). Applying a linear model to both modulator loops, the outputs from the loops can be obtained:

$D_{a}(z)=V_{i n}+N T F_{a}(z)\left(Q_{a}-z^{-1} Q_{b}\right)$

$D_{b}(z)=V_{i n}+N T F_{b}(z)\left(Q_{b}-z^{-1} Q_{a}\right)$

Assuming the NTFs of both modulators are equal, the output signal of the whole structure can be written as:

$$
\begin{aligned}
D_{a v}(z) & =\frac{D_{a}+D_{b}}{2} \\
& =V_{i n}(z)+\left(1-z^{-1}\right) N T F(z) \frac{Q_{a}(z)+Q_{b}(z)}{2}
\end{aligned}
$$

The noise shaping now exhibits an additional differentiation $\left(1-z^{-1}\right)$. Hence the effective noise shaping is of order $n+1$. The injected quantization noise is uncorrelated with the signals in the receiving loop, and acts as a dither signal. Dithering reduces the harmonic distortion and as such improves the dynamic range.

\section{Modulator Architecture}

In [8] the optimal system level design for a cross noisecoupled $2^{\text {nd }}$ order double-sampling $\Sigma \Delta$-modulator with 


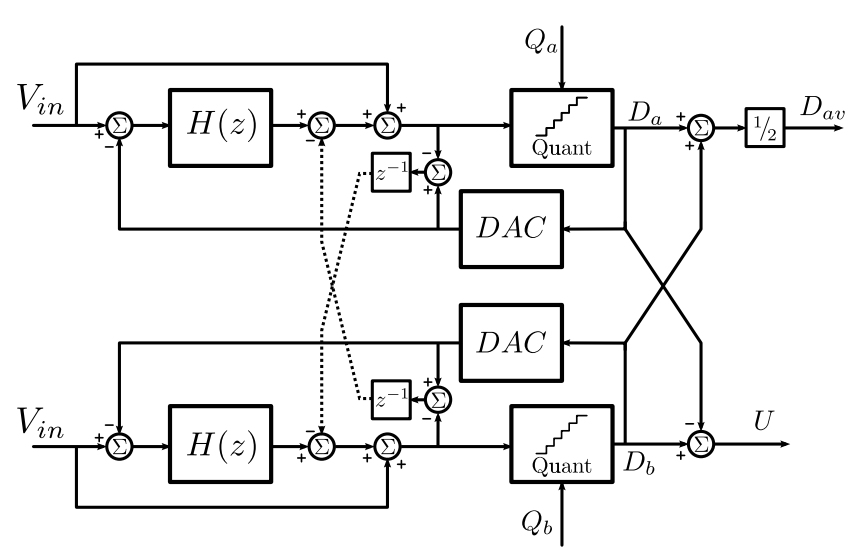

Fig. 4 A split $\Sigma \Delta$-modulator with cross-coupled noise injection.

bilinear integrators is discussed. It is shown that a good choice for the modulator parameters is:

$b_{1}=\frac{1}{2}, b_{2}=\frac{3}{2}, b_{3}=\frac{1}{2}$

The final modulator architecture is shown in Fig. 5. The loop filter $(H(z)$ in Fig. 4) consists of a cascade of two integrators with distributed feedback, similar to Fig. 3. Local feedback around the two integrators is used to optimize the NTF zeros. Additional feedin paths for the input signal $V_{i n}$ towards internal nodes of the loop filter reduce the output swing of the integrators and as such relaxes the requirements on the operational amplifier design [8]. The feedback branches for the output signal are implemented with a fully-floating bilinear integrator, while the input signal $V_{i n}$ is sampled with a conventional (non-floating) bilinear input branch. The coefficient $b_{3}$ is implemented as a feed-forward branch at the $2^{\text {nd }}$ integrator (hence the factor $\left(1-z^{-1}\right)$ ).

For a 7-level quantizer and an OSR of 20, system level simulations show that a signal to quantization noise ratio (SQNR) of $85 \mathrm{~dB}$ can be reached.

\section{Prototype Design}

The modulator is integrated in a $130 \mathrm{~nm}$ CMOS process with a supply voltage of $1.2 \mathrm{~V}$. In this technology, 2 different types of MOS-transistors are available, highspeed (HS) and low-leakage (LL). The LL-devices show a larger threshold voltage, lower leakage current and a larger gain for a single stage.

All switches are implemented with HS-transistors. For the input-sampling switch a standard CMOS transmission gate is used and the total input-sampling capacitance is $600 \mathrm{fF}$.

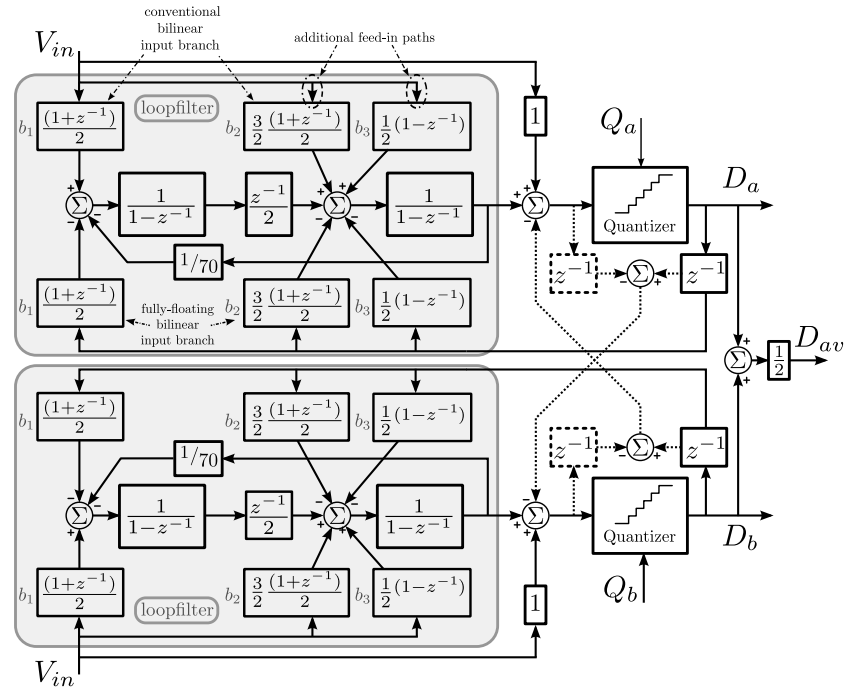

Fig. 5 The implemented modulator architecture.

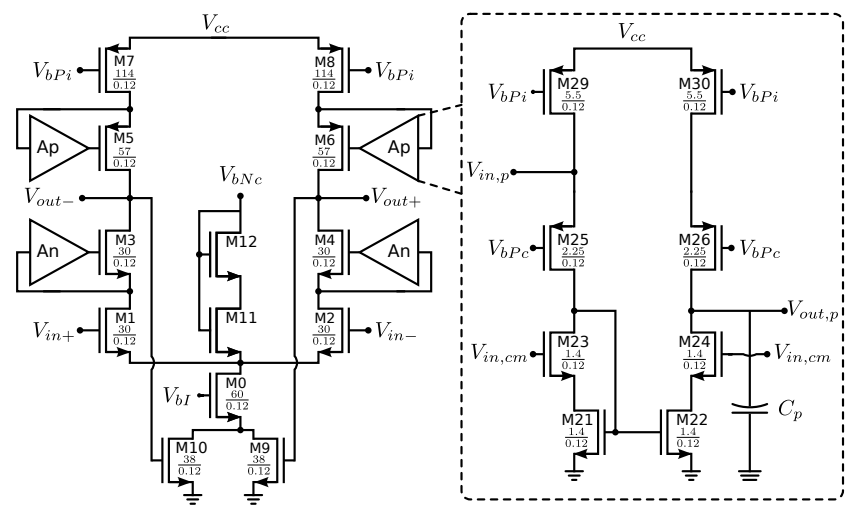

Fig. 6 The circuit for the operational amplifier used in the integrators.

\subsection{Operational Amplifiers}

The operational amplifier used for the integrators is shown in Fig. 6. In this op-amp circuit all transistors are LL-devices, except for M9 and M10, which are HStransistors. Operating in triode region, M9-M10 regulate the output common-mode voltage by adjusting the bias current through M0. Because of the low output swing of the integrators, a telescopic cascode op-amp is chosen [8]. System level simulations showed that a DC-gain of at least $55 \mathrm{~dB}$ was necessary. To achieve this high gain the cascode transistors are boosted with a high-swing low-voltage regulation amplifier [9]. The booster for the PMOS cascode is also shown in Fig. 6 . To attain an accurate settling behaviour, the boosters are band limited by adding small capacitors in their outputs. 


\subsection{Active Adder}

For the implementation of the quantization noise coupling, an active adder is needed at the quantizer input. Fig. 7(a) shows a straightforward double-sampling SCimplementation for this adder. To simplify the figure, only the input branches of the adder that implement the noise-coupling are shown, the analog input of the quantizer of the other loop $\left(V_{Q i n}\right)$ and the digital output of the other loop $\left(D=\sum d_{i}\right)$. All capacitors shown in this figure have the same capacitance and the indices $a$ and $b$ represent the two different modulator loops. Each op-amp has two input branches and two feedback capacitors with altered clock phases $\left(\phi_{1}\right.$ and $\left.\phi_{2}\right)$. Capacitor $C_{2, a}$ samples the input of the quantizer of the other modulator loop when $\phi_{1}$ is active. During the next active phase of $\phi_{2}, C_{2, a}$ is switched between the adder-input and the output of the quantizer of the other loop, $D_{b}=\sum d_{i, b}$. The net result on the feedback capacitor $C_{F B 2, a}$ is $z^{-1}\left(D_{b}-V_{Q i n, b}\right)=z^{-1} Q_{b}$. During $\phi_{1}, C_{F B 2, a}$ is reset to ensure the correct operation of the adder. $C_{1, a}$ and $C_{F B 1, a}$ perform the same operation with altered clock phases.

An improved circuit that performs the same summation is shown in Fig. 7(b). In stead of resetting the feedback capacitor $C_{F B 1}$, the same capacitor is used as feedback capacitor in the adder of the other modulator loop during the next clock phase. Since the voltage stored on $C_{F B 1}$ during $\phi_{1}$ is equal to $V_{Q i n, a}$, the feedback capacitor performs the summation of $V_{Q, i n 1}$ at the input of the quantizer in the other modulator loop. This way the capacitive load of the op-amp is halved.

For the active adder a folded cascode op-amp is used to attain the differential full-scale of $\pm 600 \mathrm{mV}$ at the input of the quantizer. Gain boosting and common mode feedback are done in a similar way as in the telescopic op-amp shown in Fig. 6.

\subsection{SC-implementation}

The SC-circuit for one modulator loop is shown in fig.8. The dashed lines implement the coupling between the 2 modulator loops. Digital signal busses are drawn with a thick line. The digital output $D$ of the loop is converted by a CMOS selection logic into selection signals for the switches implementing the D/A-conversion. The selection signals for $b_{3}$ are different from the selection signals for $b_{1}$ and $b_{2}$, as $b_{3}$ is implemented as a feed forward branch, while $b_{1}$ and $b_{2}$ are bilinear input branches

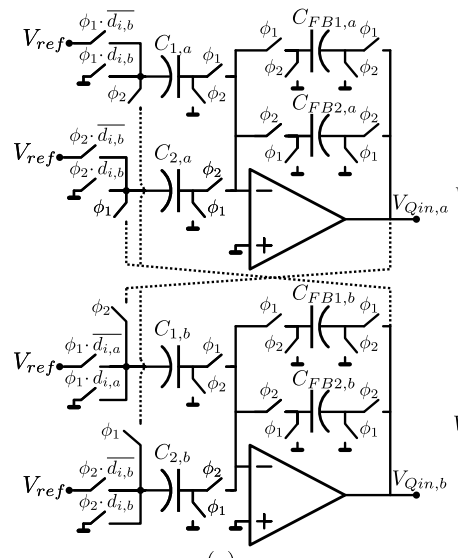

(a)
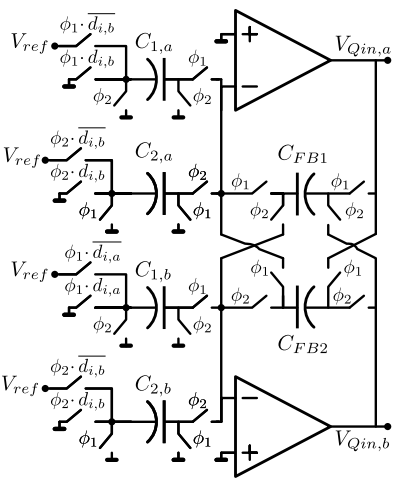

(b)

Fig. 7 (a) A double sampling adder circuit and (b) an adder with reduced load capacitance.

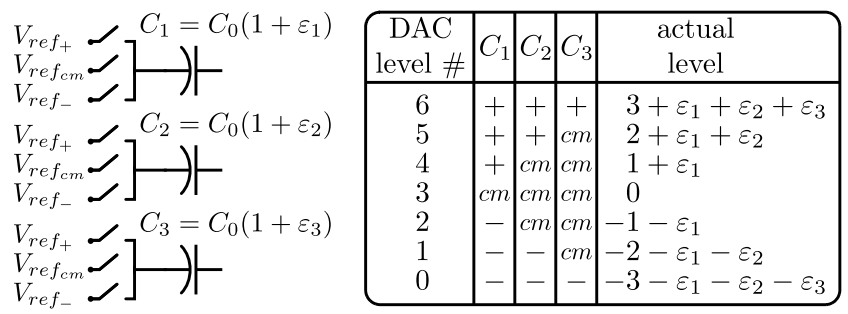

Fig. 9 Mismatch of the DAC-capacitors and the corresponding DAC-levels.

\subsection{DAC calibration}

Mismatch in the DAC capacitors is unavoidable. Due to this, the actual DAC level will deviate from its nominal value. This phenomenon is illustrated in Fig. 9. As a result, the relationship between the digital input value of the DAC and the corresponding output level will be non-linear. Since this DAC is used in the feedback path of our $\Sigma \Delta$ modulation ADC, this capacitor mismatch will in the end lead to ADC distortion [10]. A traditional way to solve this problem is to linearize the feedback DAC by the use of a dynamic element matching (DEM) [10] technique. Here, Data-WeightedAveraging (DWA) is the simplest (and most popular) variant. However, in our double-sampled modulator, this technique is nearly impossible to implement due to the stringent time budget. Indeed the quantizer is strobed at the falling edge of each clock phase, while the DAC is updated at the rising edge of the following clock phase. This way the DWA selection signal should be calculated within the non-overlap between the two clock phases. Obviously, this is almost unfeasible.

Another way to counteract the non-linearity of the DAC capacitors is to use digital calibration [11]. Here the actual DAC output levels are stored in a digital look-up table (LUT). During the normal operation, the 


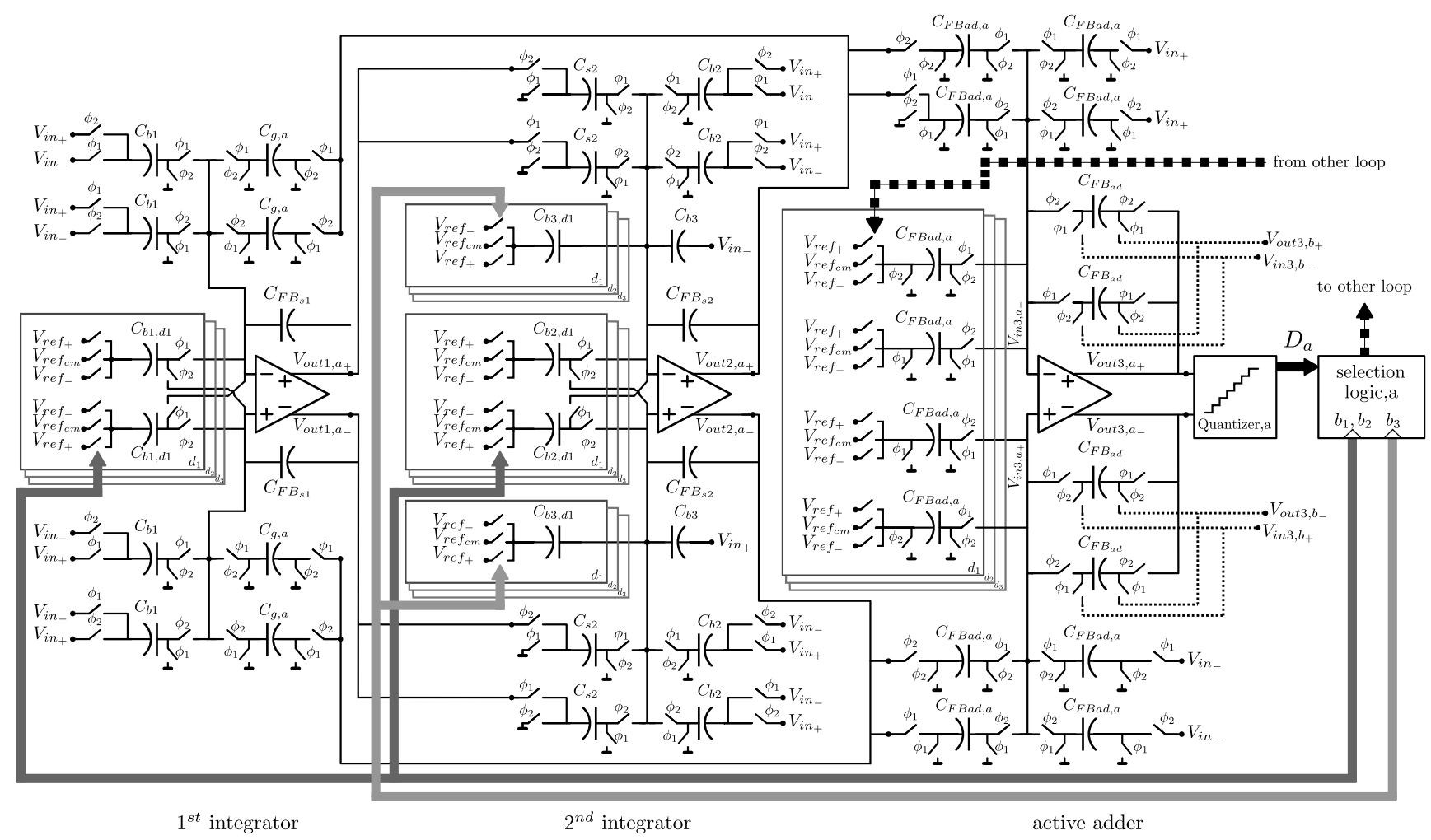

Fig. 8 SC-implementation of the modulator architecture.

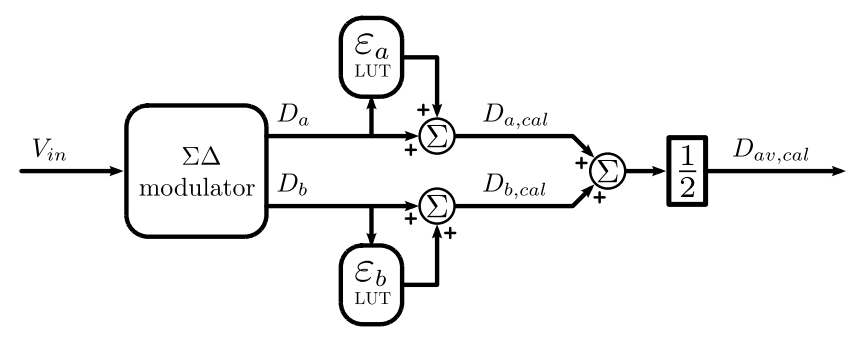

Fig. 10 The calibration scheme.

calibrated output $D_{\text {cal }}$ is obtained from the look-uptable. In our case of a fully differential 7-level DAC this look-up table contains three error terms. Since there are two feedback DAC's in our split $\Sigma \Delta$ ADC, we need two of such look-up tables. The resulting structure is shown in Fig. 10. It is clear that this calibration setup corresponds to a nearly negligible amount of additional hardware. Moreover, the calibration is not in a timing critical signal path and hence is not subject to particular timing constraints. In our prototype the look-up table is not integrated in the chip and implemented in software.

Obviously, the accuracy of such a calibration depends on how accurately the values in the look-up table correspond to the actual DAC-errors. In this prototype, the values for the look-up table are first determined in an offline LMS calibration cycle (which is further out of the scope of this manuscript). Then the calibration values are stored in memory for use during the normal operation (shown in Fig. 10). Clearly, this approach cannot correct drifting due to e.g. thermal effects or aging. If this is expected to be a problem, techniques for continuous (online) calibration such as [12] can be used. Such techniques can also successfully correct drifting DAC values, but this was not considered necessary in our case of a switched capacitor DAC and hence no further attention was given to this option.

\section{Measurement Results}

A prototype that incorporates the above described techniques was designed and submitted for fabrication. Unfortunately, the first silicon samples that we received were subject to a fabrication error. Due to this fabrication error, all the transistors were out of spec. As a result, sampling switches and clock drivers suffered from inadequate drive strength. Still these first samples were fully functional $\Sigma \Delta$ ADC's (be it at a reduced speed and with increased distortion) and their performance was reported in [13]. The measurements reported in this manuscript are from a second batch of samples which is fully compliant now.

Fig. 11 shows a microscope photograph of the prototype chip. Fig. 12 shows a measured spectrum (21K 


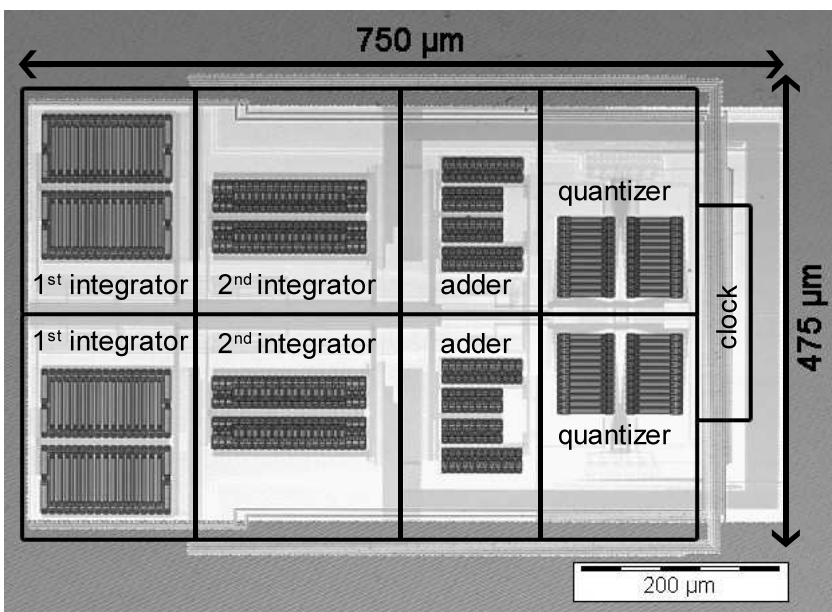

Fig. 11 Microscope photograph of the integrated $\Sigma \Delta$-ADC.

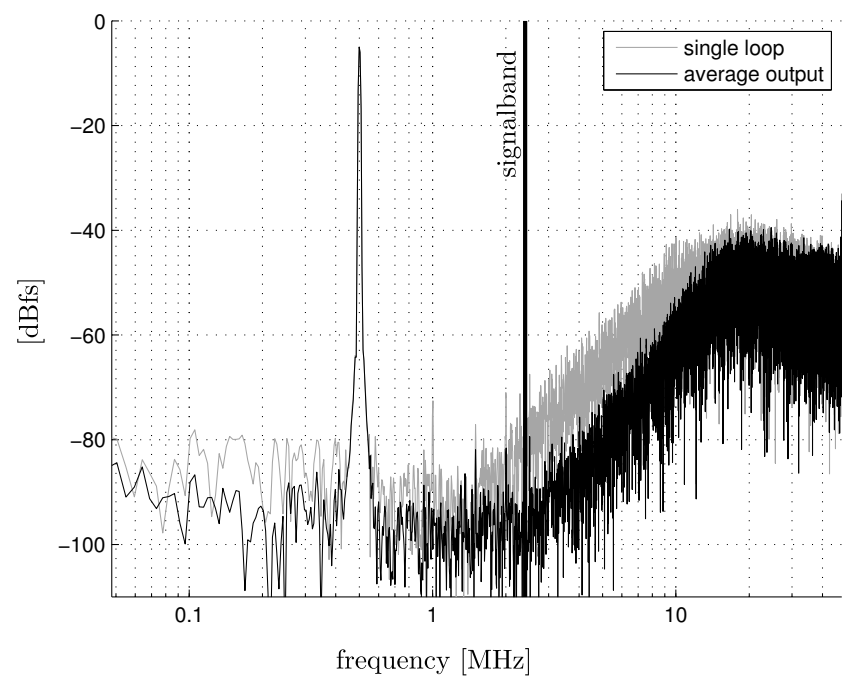

Fig. 12 Measured output spectrum for a -5 dBFS $501 \mathrm{kHz}$ input tone.

FFT - blackman window) for a sinusoidal input signal of $501 \mathrm{kHz}$ and a $-5 \mathrm{~dB}$ amplitude relative to fullscale (dBFS) after calibration. The greyed spectrum corresponds to a single modulator, whereas the black spectrum corresponds to combined spectrum of both coupled modulators. It is clear that the noise-coupling works as expected and greatly enhances the noise shaping. For this measurement, the 3rd harmonic is at $-82.4 \mathrm{~dB}$ and the 4 th harmonic is at $-82 \mathrm{~dB}$. Fig. 13 shows the SNR and SNDR as a function of input signal amplitude (measured at $501 \mathrm{kHz}$ ). The peak SNR is $67.8 \mathrm{~dB}$ for $2.2 \mathrm{dBFS}$ and the peak SNDR is $66.1 \mathrm{~dB}$ for $-3 \mathrm{dBFS}$. The dynamic range is $72 \mathrm{~dB}$.

The previous measurements were for the case where the digital calibration of Fig. 10 is active. The effect of the digital calibration is illustrated in Fig. 14, where measured spectra with and without digital calibration are shown. Here the signal frequency is chosen such

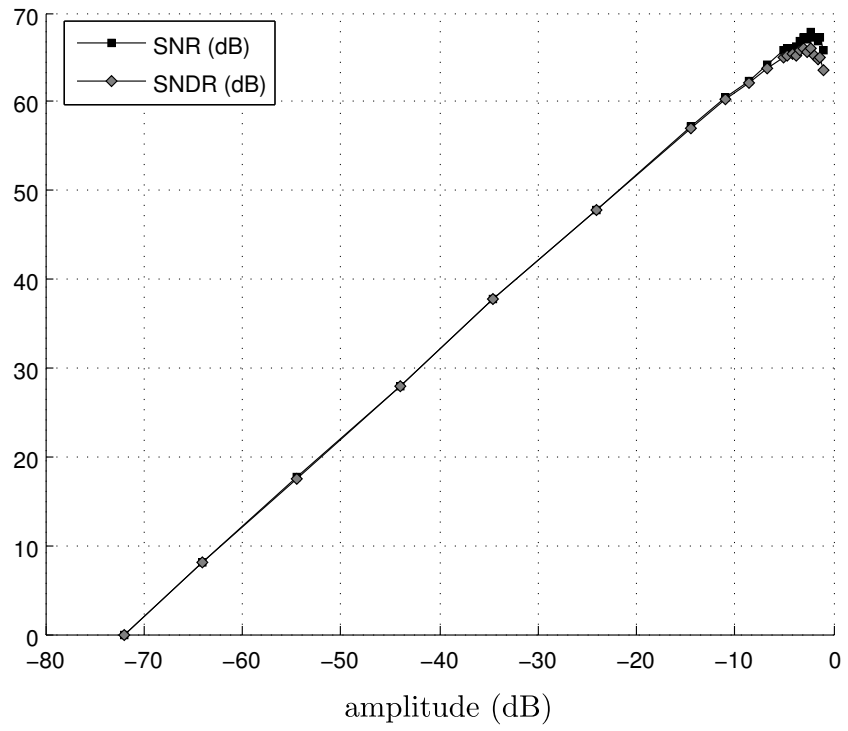

Fig. 13 SNR and SNDR vs. input amplitude for a $501 \mathrm{kHz}$ input tone.

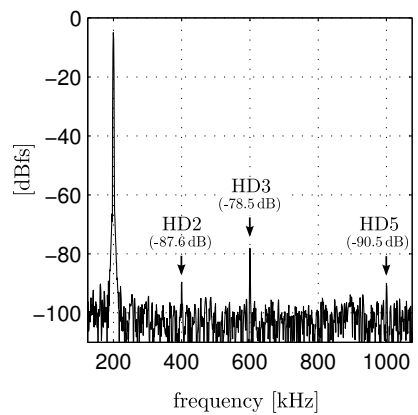

(a)

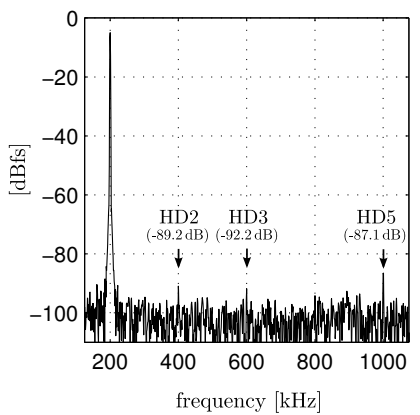

(b)
Fig. 14 Measured output spectrum for a -5 dBFS $201 \mathrm{kHz}$ input tone without (a) and with (b) calibration.

that the 5th harmonic is still within the signal band. In the case without digital calibration (Fig. 10.a) the SFDR is limited by the 3rd harmonic. After calibration (Fig. 10.b) the SFDR improves by $9 \mathrm{~dB}$ and is now limited by the 5 th harmonic which was slightly increased. The resulting distortion is attributed to signal dependent charge injection in the sampling switches.

The high frequency linearity is illustrated in Fig. 15, where the measured spectrum for a two-tone test is shown. Here the two tones are placed near the band edge. For this measurement the digital calibration is activated. From the magnitude of the intermodulation products we can conclude that the distortion increases for higher input frequencies which could indicates that there is tracking distortion in the sampling switches.

The total power dissipation is $8 \mathrm{~mW}$ of which $6.4 \mathrm{~mW}$ is dissipated in the analog core. Fig. 16 shows a detailed distribution of the power consumption. From the above measurements we can also calculate Walden's figureof-merit (FOM) [14], defined by $F O M=P /(2 \cdot B W$. 


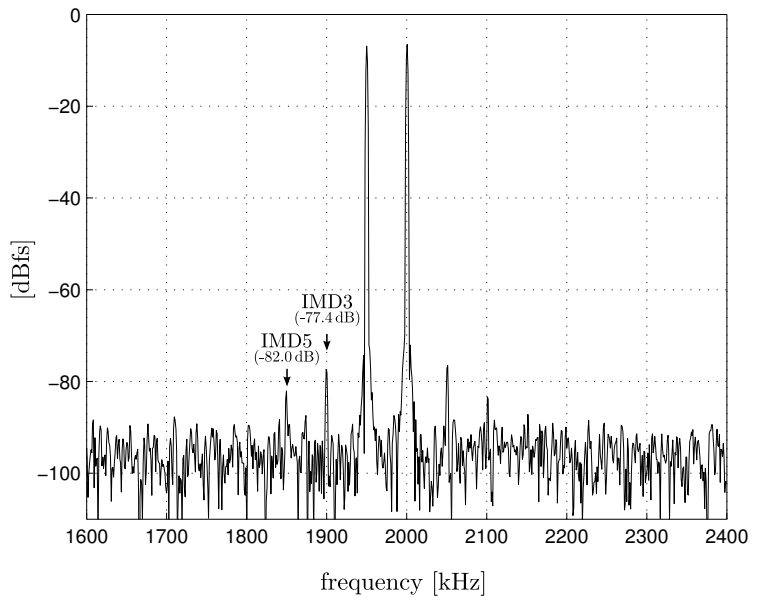

Fig. 15 Measured output spectrum for two $-7 \mathrm{dBFS}$ $1.95 \mathrm{MHz}$ and $2 \mathrm{MHz}$ input tones.

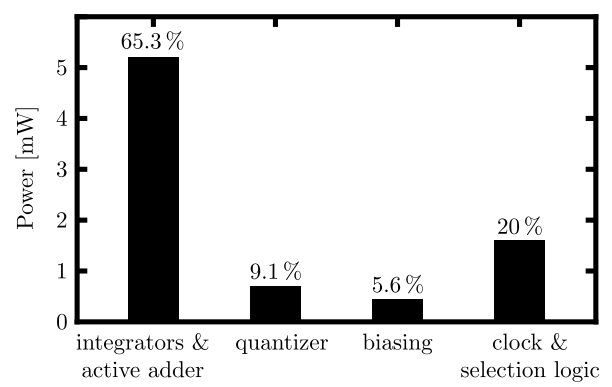

Fig. 16 Power consumption distribution.

$\left.2^{E N O B}\right)([14])$, as $0.5 \mathrm{pJ} /$ conversion-step. It should be noted though, that the relevance of this FOM has been subject to debate [15] and that it is only added here for illustrative purposes.

These measurement results, as well as a comparison with other recent switched-capacitor $\Sigma \Delta$ ADCs are summarized in table 1 . From the table it is clear that our prototypes performance compares well with other recent implementations.

\section{Conclusion}

A split $\Sigma \Delta$-A/D converter architecture combining quantization noise-coupling and double-sampling is presented. By adding cross noise-coupling between the two identical loops of the split second order modulator, the effective noise shaping order is increased to 3 . To tackle the problem of quantization noise folding in a doublesampling SC-circuit, a fully floating bilinear input branch is used for the feedback of the $\mathrm{D} / \mathrm{A}$-converter. A prototype achieves $72 \mathrm{~dB} \mathrm{DR}$ and $68 \mathrm{~dB} \mathrm{SNR}$ over a $2.4 \mathrm{MHz}$ signal band for a power budget of $8 \mathrm{~mW}$.

\section{Acknowledgement}

This work was supported by the Flemish Fund for Scientific Research (FWO-Vlaanderen).

\section{References}

1. Lee, K., Temes, G.C. (2006). Enhanced split-architecture $\Sigma \Delta$ ADC. Electronic Letters, 42(13), 737-739.

2. Lee, K., Bonu, M., Temes, G.C. (2006). Noise-coupled $\Sigma \Delta$ ADCs. Electronic Letters, 42(24), 1381-1382.

3. Bilhan, E., Maloberti, F. (2009). A Wideband Sigma-Delta Modulator With Cross-Coupled Two-Paths. IEEE Transactions on Circuits and Systems-I, 56(5), 886-893.

4. Silva, J., Moon, U.,Steensgaard, J.,Temes, G.C. (2001), Wideband low-distortion delta-sigma ADC topology. Electronic Letters, 37(12), 737-738.

5. Rombouts, P., Raman, J., Weyten, L. (2003). An approach to Tackle Quantization Noise Folding in Double-Sampling $\Sigma \Delta$ Modulation A/D Converters. IEEE Transactions on Circuits and Systems-II, 50(4), 157-163.

6. Nagari, A., Mecchia, A., Viani, E., Pernici, S., Confalonieri, P., Nicollini, G. (2000). A 2.7-V 11.8-mW Baseband ADC With 72-dB Dynamic Range for GSM Applications. IEEE Journal of Solid-State Circuits, 35(6), 798-806.

7. Rombouts, P., De Maeyer, J., Weyten, L. (2005). Design of Double-Sampling $\Sigma \Delta$ Modulation A/D Converters With Bilinear Integrators. IEEE Transactions on Circuits and Systems-I, 52(4), 715-722.

8. De Bock, M., Rombouts, P. (2009). A double-sampling cross noise-coupled split $\Sigma \Delta$ A/D converter with $80 \mathrm{~dB}$ SNR. 16th IEEE International Conference on Electronics, Circuits, and Systems, 45-48.

9. Sumanen, L., Waltari, M., Halonen, K.A.I. (2001). A 10bit 200-MS/s CMOS parallel pipeline A/D converter. IEEE Journal of Solid-State Circuits, 36(7),1048-1055.

10. Schreier, R., Temes, G.C. (2005). Understanding DeltaSigma Data Converters: Implementation Considerations For $\Delta \Sigma$ ADCs (pp. 179-218). New York: Wiley.

11. Sarhangnejad, M., Temes, G.C. (1993), A high-resolution multibit-Sigma-Delta ADC with digital correction and relaxed amplifier requirements, IEEE Journal of Solid-State Circuits, 28(6), 648-660.

12. Witte, P., Ortmanns, M. (2010). Background DAC Error Estimation Using a Pseudo Random Noise Based Correlation Technique for Sigma-Delta Analog-to-Digital Converters. IEEE Transactions on Circuits and Systems-I, 57(7), 15001512

13. De Bock, M., Rombouts, P. (2010). A $5.8 \mathrm{~mW} 72 \mathrm{~dB} \Sigma \Delta$ modulator ADC with $1.8 \mathrm{MHz}$ BW in $130 \mathrm{~nm}$ CMOS. IEEJ Analog VLSI Workshop Proceedings, 87-90.

14. Walden, R. (1999), Analog-to-digital converter survey and analysis. IEEE Journal on Selected Areas in Communication, 17(4), 539-550.

15. Murmann, B. (2008), A/D Converter Trends: Power Dissipation, Scaling and Digitally Assisted Architectures. IEEE Custom Integrated Circuits Conference Proceedings, $105-112$

16. Nam, K., Lee, S., Su, D., Wooley, B. (2005). A lowvoltage low-power sigma-delta modulator for broadband analog-to-digital conversion. IEEE Journal of Solid-State Circuits, 40(9), 1855-1864.

17. Ranjbar, M., Mehrabi, A., Oliaei, O., Carrez, F. (2010). A $3.1 \mathrm{~mW}$ Continuous-Time Delta Sigma Modulator With 5-Bit Successive Approximation Quantizer for WCDMA. IEEE Journal of Solid-State Circuits, 45(8), 1479-1491. 
Table 1 Performance Comparison

\begin{tabular}{c|cccccccc} 
Feature & This Work & {$[16]$} & {$[17]$} & {$[18]$} & {$[19]-\mathrm{A}$} & {$[19]-\mathrm{B}$} & {$[20]$} & {$[21]$} \\
\hline CMOS technology (nm) & 130 & 250 & 130 & 180 & 180 & 180 & 180 & 90 \\
Supply voltage (V) & 1.2 & 1.2 & 1.2 & 1.2 & 24 & 24 & 1.8 & 1.2 \\
Oversampling ratio & 20 & 16 & 48 & 16 & 1.5 & 1.5 & 8 & 20 \\
Signal bandwidth (MHz) & 2.4 & 1.25 & 1.92 & 0.625 & 4.2 & 2.5 & 6 & 1.92 \\
Peak SNDR (dB) & 66.1 & 89 & 59 & 74.6 & 79 & 81 & 60.7 & 65.5 \\
Dynamic range (dB) & 72 & 96 & 62 & 77 & 81 & 83 & 62 & 66 \\
Active area (mm2) & 0.36 & 8.6 & 0.36 & 1.92 & 3.67 & 3.67 & 0.32 & 0.076 \\
Power consumption (mW) & 8 & 44 & 3.1 & 3.2 & 28 & 15 & 6.18 & 6.8 \\
FOM [14] (pJ/conv-st) & 0.5 & 0.64 & 0.788 & 0.58 & 0.48 & 0.33 & 0.58 & 1.17 \\
\hline
\end{tabular}

18. power Maghari, N., Kwon, S., Moon, U.-K. (2009). 74 dB SNDR Multi-Loop Sturdy-MASH Delta-Sigma Modulator Using $35 \mathrm{~dB}$ Open-Loop Opamp Gain. IEEE Journal of Solid-State Circuits, 44(8), 2212-2221.

19. Lee, K., Chae, J., Aniya, M., Hamashita, K., Takasuka, K., Takeuchi, S., Temes, G. C. (2008). A NoiseCoupled Time-Interleaved Delta-Sigma ADC With 4.2 MHz Bandwidth,-98 dB THD, and $79 \mathrm{~dB}$ SNDR, IEEE Journal of Solid-State Circuits, 43(12), 2601-2612.

20. Bonizzoni, E., Perez, A. P., Maloberti, F., GarciaAndrade, M. A. (2011). Two op-amps third-order sigmadelta modulator with 61-dB SNDR, 6-MHz bandwidth and 6-mW power consumption, Analog Integrated Circuits and Signal Processing Journal, 66(3), 381-388.

21. Bos, L., Vandersteen, G., Rombouts, P., Geis, A., Morgado, A., Rolain, Y., Van der Plas, G. and Ryckaert, J. (2010). Multirate Cascaded Discrete-Time Low-Pass $\Delta \Sigma$ Modulator for GSM/Bluetooth/UMTS, IEEE Journal of Solid-State Circuits, 45(6), 1198-1208. 